\title{
The importance of accurate water metering in resource management
}

\author{
M. B. Salamah ${ }^{1}$, A. Kapoor ${ }^{2}$, M. Savsar ${ }^{3}$, \\ M. Ektesabi ${ }^{2}$, \& A. Abdekhodaee ${ }^{2}$ \\ ${ }^{1}$ The Electric Power Section of the Higher Institute for Energy, \\ The Public Authority for Applied Education and Training (PAAET), \\ Kuwait \\ ${ }^{2}$ The Faculty of Engineering, Swinburne University of Technology, \\ Melbourne, Australia \\ ${ }^{3}$ The Industrial and Management Systems Engineering Section, \\ The College of Engineering \& Petroleum, Kuwait University, Kuwait
}

\begin{abstract}
Water has always been the source of growth and dispute between providers and consumers. Nowadays, water conservation and environmentally friendly technologies are becoming more important, especially in areas where access to water is expensive or restricted. Since water has become a limiting resource for economic development, accurate measurement of consumption is important for both trade and industry. Unfortunately, over time and use, the internal components of a measuring flow meter wear, and the level of metering accuracy drops. This progressive drop in metering accuracy causes "meter drift".

This paper studies the effect of drift/errors in flow meters used in seawater pumping stations, which are supplying seawater for several refineries and petrochemical plants in Kuwait. The seawater pumped to these consumers is utilised for cooling and process purposes. In such high volume consumer system, a small drift may result in lost revenue due to an under-accounting of the quantity of used water. In such systems, in order to endlessly repair, rebuild or recalibrate all the flow meters a labour intensive and expensive process is required that causes significant down-time too. In the presented research work, a novel mathematical method is presented which provides early detection of flowmeter drift irrespective of type (ultrasonic, magnetic, etc.). This method is designed based on the mathematical models of the system and its process relations. The proposed method works with minimal input data and provides an inexpensive solution to existing measuring systems.
\end{abstract}

Keywords: flow meter, flow meter drift, data analysis, statistical process control (SPC). 


\section{Introduction}

This research was conducted at an organization that owns seawater pumping stations, supplying seawater for several refineries and petrochemical plants in Kuwait. The seawater pumped to these consumers is utilized for cooling and process purposes. The quantity of seawater supplied is measured by a flow meter (or flowmeter). The income generated by the pumping stations is around US\$32 million a year, about half of the revenues of the mother organization. In this case, a small error in reading will be very costly. On the other hand, the flow meters are susceptible to errors and inaccurate readings. For higher accuracy and to avoid the errors, calibration is needed which involves checking of each measuring instrument against accurate standards to determine any deviation and correct for errors. This process is time consuming and very costly.

In this plant, maintenance in general and calibration specially is done as a matter of priority. A very critical device related to cases that may impose danger to the life of the workers has highest priority. Maintenance and calibration of the pumping station devices that are critical to production to ensure meeting contractual obligations to the consumers for continuous and undisturbed supply of seawater is the second priority because, any interruption of seawater supply to a consumer would incur a heavy cost. Consumption flow meters are neither life nor production critical. This would make them in the category of devices not receiving much attention. Indeed, they are usually not thought of except once every month at billing time.

There are many things that can go wrong in a flow meter: its chamber might be flooded by rain or underground water causing damage or its cables might get electrically grounded causing problems in the electrical grid of the station. What we are concerned with, however, are the faults that have to do with the accuracy of its readings. Therefore, in this research, we define a flow meter fault as giving inaccurate readings. Accordingly, a fault may be salient such as what will happen when a flow meter gives a reading that the plant operators know is far above or below what a particular consumer will take. On the other hand, a flow meter fault might be hidden. This will happen when a flow meter incrementally but systematically gives inaccurate readings that would go unnoticed by the operation staff. This is known as flow meter drift. The detection of the later type of flow meter fault (flow meter drift) is the subject of this research.

It is doubtful that an industrial consumer will accept to pay in retrospect because the inaccuracy of the flow meter is the fault of the pumping station. Therefore, it is extremely important to detect flow meter inaccuracy as fast as possible in order for the pump station owners to calibrate it and avoid any financial loss.

Nilsson [1] has mentioned that inaccurate measurement is responsible for more than $80 \%$ of the unaccounted-for loss in gas supply industry. A similar phenomenon, unaccounted-for water loss, is known in the potable water industry have been reported [2,3]. From the researchers' own experience, the phenomenon exists in the cooling water industry also. In fact, the issue of flow meter inaccuracy is a problem wherever flow meters are used regardless of the 
fluid being measured. For example, a flow meter error that resulted in unaccounted-for loss in petroleum caused a political crisis in Kuwait. All of these problems that have to do with flow meter inaccuracy are just a part of a greater problem which is the accuracy of the measuring instruments in general.

Not surprisingly, the issue of flow meter accuracy was the subject of many research papers and patents. However, the majority of these have been about improving the sensing devices and electronic circuitry [4,5]. Therefore, the immediate benefits of such works are for flow meter manufacturers. Big industrial users, on the other hand, cannot afford to change the meters as of technology improvements. The change, modifications and replacement are very costly practice for such industries. In addition, making a continuous online flow meter monitoring system would also be both complicated and expensive. Thus, an alternative method of improving flow meter accuracy and detecting inaccurate measurement was proposed here. The alternative method could be by observed measured pattern of consumption. Any deviation from the usual pattern is considered an indication of possible inaccurate reading (fault) that would require calibration. This made the alternative approach some sort of statistical methods to be devised. The use of statistical methods for the detection of flow meter inaccuracy was thought of as an attractive option for many reasons including

- It did not involve tampering with flow meter circuitry and the risks involved in such an option.

- It was a universal solution independent of the flow meter type (electromagnetic, ultrasonic...etc.) or manufacturer.

- It was capable to work with the data from the monthly bills i.e. it needed minimal data.

- It did not need any knowledge about the mathematical relations and models that governed the process.

- It was inexpensive.

In this paper, when any deviation from normal consumption pattern was identified, it is considered as an indication of a possible flow meter fault that would alarm requirement of the Instrument Section to investigate and calibrate the flow meter in question to check for its accuracy. In the following section the use of statistical methods for the detection of flow meter inaccuracy is presented.

\section{SPC and CUSUM techniques}

Statistical process control (SPC) is a method for monitoring the mean of a process and is a very popular method. Han and Tsung [6] ascertain that the current methods of SPC focus mostly on monitoring and detection of constant shifts in the mean and other conditions where the mean shift is dynamic have not been thoroughly studied. To fill this gap, Han and Tsung [6] designed a chart for tracing and detecting dynamic mean changes.

The determining work on Cumulative Sum (CUSUM) reported by Page [7] and recently improvements of this is proposed by Zhao et al [8] who produced a dual CUSUM (DCUSUM) by combining two CUSUM charts to detect a range of mean shifts. Han et al [9] stated that although the CUSUM method is simple, 
its performance deteriorates when the actual mean shift is unknown. To overcome this condition, an alternative approach called the CUSUM chart with local signal amplification (LSA-CUSUM) was presented. This scheme worked by amplifying or weakening local signals to improve the power of the traditional CUSUM chart in detecting an unknown mean shift over a range. Han et al claimed that measurable weakening and amplifications of local signals can improve the ability of the CUSUM chart in detecting the local mean shift. Han et al [10] also created a multi chart consisting of several CUSUM or EWMA charts with different reference values that are used simultaneously to detect anticipated process changes. Their work shows that the multi-chart has the merits of quick detection of a range of mean shifts, was easy and had a flexible design for various situations and great reduction in computational complexity. Han and Tsung [11] also made a CUSUM multi-chart scheme consisting of multiple CUSUM control charts for detecting and diagnosing unknown abrupt changes in a process. They showed that this scheme performed better than the single CUSUM or EWMA, CUSUM-multi chart, EWMA-multi chart and GLR (Generalized Likelihood Ratio) charts.

Of the many statistical methods available, statistical process control (SPC) was thought as the most appropriate one. Also, of the many SPC methods existing, the tabular Cumulative Sum (CUSUM) was thought of as the most suitable method for detecting flow meter drift. The consumption data takes the form of a seasonal time series that widely oscillates (increasing in the summer and decreasing in the winter). This time series is not suitable for use in SPC in general and more specially in a CUSUM method. The CUSUM method is basically a method that detects mean shift. A method had to be thought of to transform the seasonal time series consumption data to a medium suitable to be used in SPC-CUSUM. This has been done by creating a virtual mean $\overline{x_{v}}$. For every point in the time series (regardless of its location) there would be a virtual point that represents the virtual mean corresponding to this point. It was this virtual mean that was processed by the CUSUM method.

\section{Implementation and comparison}

As mentioned above, seasonal time series are not the best medium for SPC in general and the tabular CUSUM specially. All SPC methods require that the process data to be closely gathered around a mean. This is not the case of a seasonal time series were data are usually very far from the mean. One of the research problems, therefore, became of finding a suitable method of transforming the seasonal time series to a linear time series. This problem is depicted in figure 1 below.

The transformation was achieved by exploiting two features of a sinusoidal wave and their corresponding features of seasonal time series. The explanations follow. 


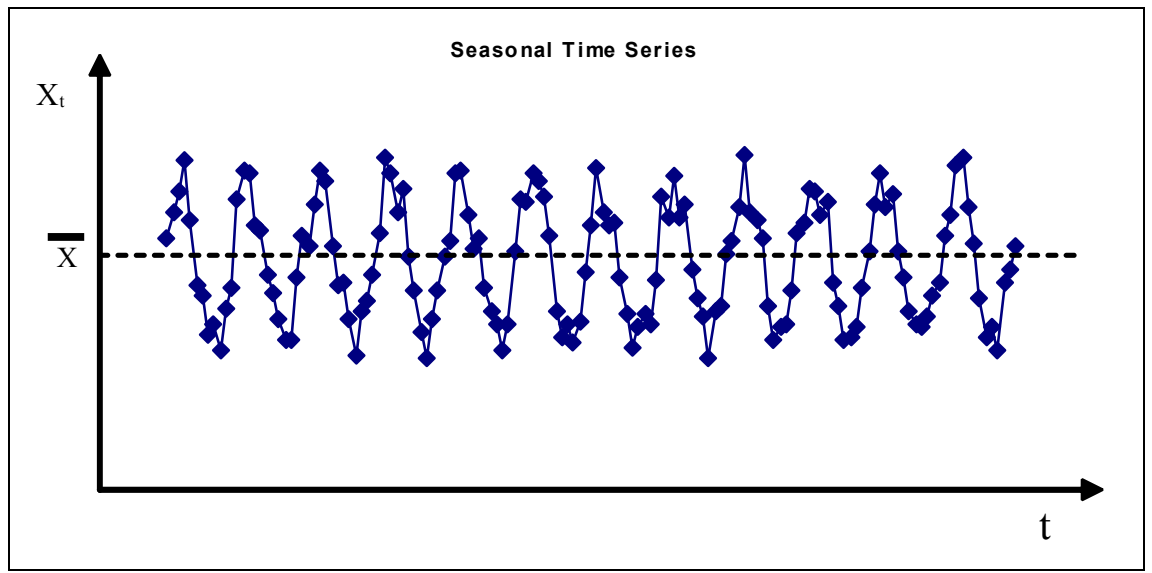

Figure 4: Seasonal time series.

In the case of a seasonal time series, the virtual or running mean for every point will be called function $f_{t}\left(\overline{\left.X_{v}\right)}\right.$ and it represents the virtual mean for every point.

$$
f_{t}\left(\overline{\left.X_{v}\right)}=X_{t}-X_{(t-d)}+\frac{X_{t}+X_{\left(t-\frac{d}{2}\right)}}{2}+\varepsilon\right.
$$

where

$$
\varepsilon=\varepsilon_{1}+\varepsilon_{2}
$$

and $\varepsilon$ is i.i.d.

The graph of the function for the virtual mean against the time series of figure 4 is represented in figure 5 .

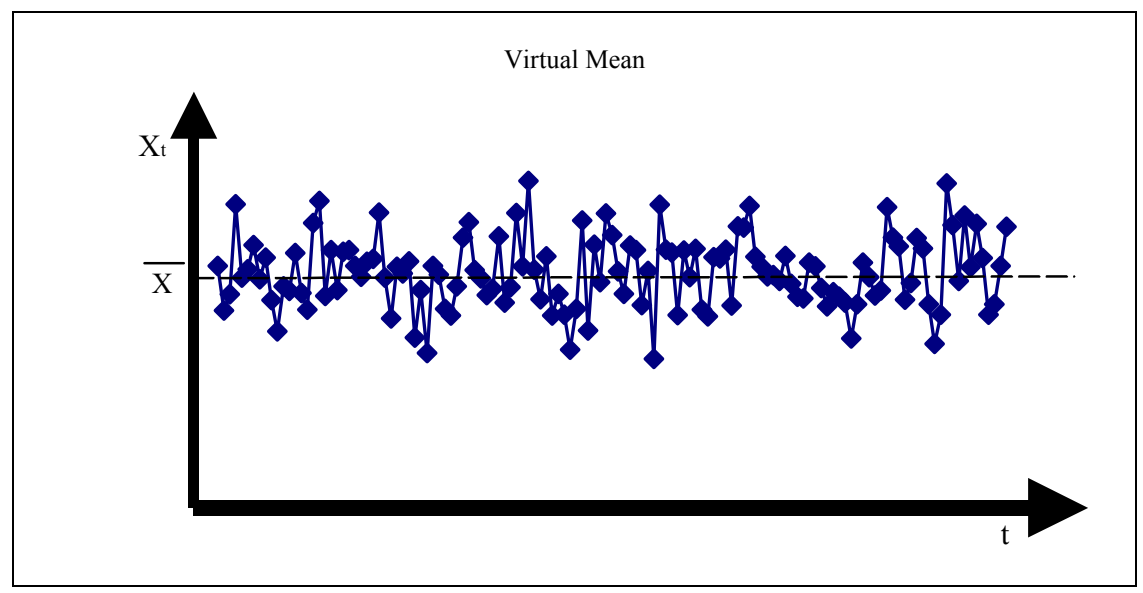

Figure 5: Virtual mean values. 
It can be shown that the running or virtual mean given in equation (9) equals the seasonal- time series' estimated mean plus some residual.

$$
f_{t}\left(\overline{X_{v}}\right)=\bar{X}+\varepsilon
$$

This is exactly what is needed for a process to be monitored by SPC methods and, more specifically, the tabular CUSUM.

\subsection{The Method of the CUSUM}

According to Montgomery [12], the cumulative sum (CUSUM) is done by plotting the cumulative sums of the deviations of the sample values from a target value, in our case the virtual mean.

$$
C_{i}=\sum_{j=1}^{i}\left(X_{i}-\bar{X}\right)
$$

Or, put in another way,

$$
C_{i}=\left(x_{i}-\bar{x}\right)+C_{i-1}
$$

where $C_{i}$ is the cumulative sum up to and including the $i^{\text {th }}$ sample with starting value $\mathrm{C}_{0}=0$.

\subsection{The method of tabular CUSUM}

According to Montgomery [12], the tabular CUSUM works by accumulating deviations from the target that are above it with one statistic $\mathrm{C}^{+}$and accumulating deviations from the target that are below it with another statistic $\mathrm{C}^{-}$. If the virtual mean is the target, then;

$$
\begin{aligned}
& C_{i}^{+}=\max \left[0, x_{i}-(\bar{x}+k)+C_{i-1}^{+}\right] \\
& C_{i}^{-}=\max \left[0,(\bar{x}-k)-x_{i}+C_{i-1}^{-}\right]
\end{aligned}
$$

where $\mathrm{C}_{0}^{+}=\mathrm{C}_{0}^{-}=0$, and $\mathrm{k}$ is a reference value; $\mathrm{k}$ is one-half the magnitude of the shift we are interested in detecting. In this paper the magnitude of the shift we are interested in detecting is one standard deviation. Accordingly,

$$
k=\frac{\sigma}{2}
$$

Note that $\mathrm{C}_{\mathrm{i}}^{+}$and $\mathrm{C}_{\mathrm{i}}^{-}$accumulate deviations from the target value (in our case the virtual mean) that are greater than $\mathrm{k}$ with both quantities being rest to zero on becoming negative. If either $\mathrm{C}_{i}^{+}$or $\mathrm{C}_{\mathrm{i}}^{-}$exceeds a decision interval, $\mathrm{H}$, the process is considered to be out of control. Montgomery suggests that a reasonable value for $\mathrm{H}$ is five times the process standard deviation.

$$
H=5 \sigma
$$

In this research paper we followed Montgomery's suggestion.

\section{Drift detection by statistical methods}

In this section, we are studying some cases of flow measurement and drift occurred in the actual plants. In this case, the reading of the flow meter of a plant "named C7" is shown in figure 6. 


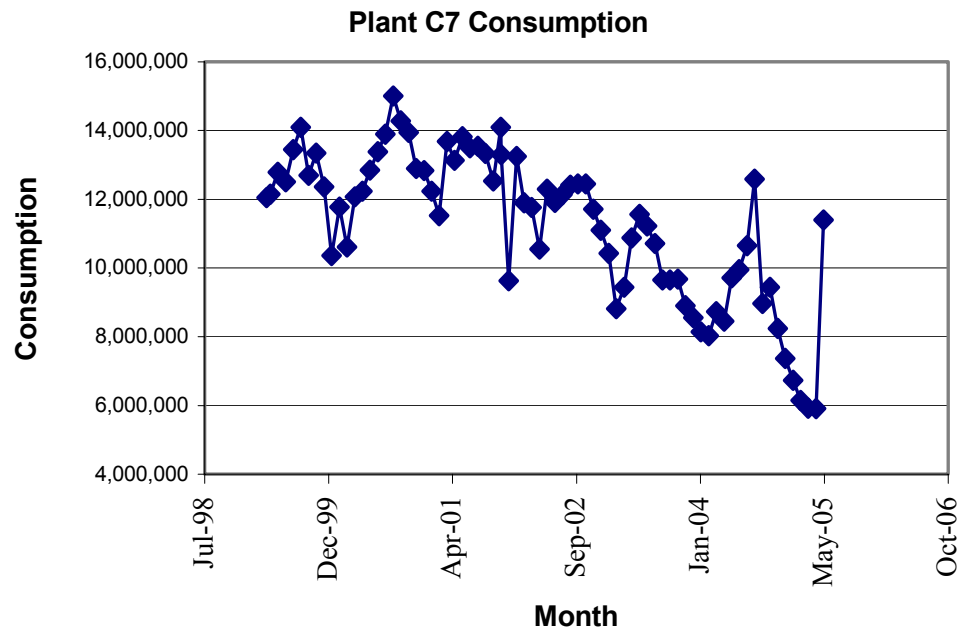

Figure 6: Flow meter readings.

It is obvious that the flow meter readings for the consumption are decreasing over the years. At that time it was thought that the decrease in consumption was due to C7's economy in the use of seawater and not to a flow meter fault.

Suspicions in the abnormality of the plant consumption only started when it was, coincidently, noticed that the pressure produced by operating pumps (for C7) should give more flow than what was recorded by the flow meter. This meant one of three possibilities

- The pressure gauges were wrong, or

- The flow meter was wrong, or

- There was a major leak in the pipeline.

The pressure gauges were calibrated and were found to be OK and there was no leak in the pipeline. This made the flow meter the only suspect. When the flow meter in question was inspected and calibrated, it became clear that it was inaccurate. This meant that $\mathrm{C} 7 \mathrm{did}$ not use less water during that period and its efforts to save water were a failure. The proof of this is the "jump" in flow meter readings after calibration (The flow meter drift was discovered in the first week of May 2005 which is the last point in the graph).

Case \#1

We investigated this application using tabular CUSUM method to verify that it would be able to do early detection of drift and to determine the time of the drift's origin. The statistical properties of the virtual mean and the parameters of the Tabular CUSUM for this specific consumer are shown in table 1, while the application of the method is shown in figure 6 .

In this case the tabular CUSUM method was able to detect the drift on December 2001. The method also indicates that the start of the drift happened some time at July 2001. What is interesting about this particular case is that the records show that a calibration took place on October 2004. It may be concluded that this calibration was not perfect. 
Table 1: $\quad$ Calculated parameters of tabular CUSUM.

\section{Case \#2}

\begin{tabular}{|l|l|}
\hline Mean $\bar{x}$ & $13,566,054$ \\
\hline Standard Deviation & 915,876 \\
\hline $\mathrm{K}$ & 457,938 \\
\hline$(\bar{x}+k)$ & $14,023,992$ \\
\hline$(\bar{x}-k)$ & $13,108,116$ \\
\hline $\mathrm{H}$ & $4,579,380$ \\
\hline
\end{tabular}

This case study is about the same consumer, C7. Another study was conducted on the period from June 2006 to September 2007. The statistical properties of the virtual mean and the parameters of the tabular CUSUM for this specific consumer on this period are shown in table 2 .

It is evident that on August 2007, the alarm persisted for three consecutive months. This might be an indication of drift. It also shows that the onset of this drift was on May 2007.

Table 2: $\quad$ Information from June 2006 to September 2007.

\begin{tabular}{|c|c|c|c|c|c|c|c|c|c|}
\hline Month & Consumption & $\mathbf{X t - ( X ( t - 1 2 ) )}$ & $\frac{\mathbf{X t}+\mathbf{X}(\mathbf{t}-\mathbf{6})}{\mathbf{2}}$ & $\mathbf{F}(\mathbf{X b a r})$ & $\mathbf{C i}+$ & $\mathbf{N}+$ & $\mathbf{C i -}$ & $\mathbf{N}-$ & Status \\
\hline Jun-06 & $11,245,300$ & $-157,100$ & $10,809,850$ & $10,652,750$ & 0 & 0 & $1,163,809$ & 1 & OK \\
\hline Jul-06 & $13,390,600$ & $-668,700$ & $11,099,200$ & $10,430,500$ & 0 & 0 & $2,549,868$ & 2 & OK \\
\hline Aug-06 & $14,045,700$ & 44,500 & $12,623,350$ & $12,667,850$ & 155,882 & 1 & $1,698,577$ & 3 & OK \\
\hline Sep-06 & $13,362,200$ & $2,621,000$ & $12,125,750$ & $14,746,750$ & $2,390,664$ & 2 & 0 & 0 & OK \\
\hline Oct-06 & $13,536,200$ & $1,055,000$ & $12,802,250$ & $13,857,250$ & $3,735,945$ & 3 & 0 & 0 & Alarm \\
\hline Nov-06 & $4,300,100$ & $-7,188,600$ & $7,772,700$ & 584,100 & 0 & 0 & 0 & 0 & OK \\
\hline Dec-06 & $10,205,300$ & $-579,100$ & $11,797,950$ & $11,218,850$ & 0 & 0 & 597,709 & 1 & OK \\
\hline Jan-07 & $11,741,000$ & $1,366,600$ & $12,893,350$ & $14,259,950$ & $1,747,982$ & 1 & 0 & 0 & OK \\
\hline Feb-07 & $10,502,700$ & $1,694,900$ & $11,932,450$ & $13,627,350$ & $2,863,364$ & 2 & 0 & 0 & OK \\
\hline Mar-07 & $11,849,200$ & 648,200 & $12,692,700$ & $13,340,900$ & $3,692,295$ & 3 & 0 & 0 & Alarm \\
\hline Apr-07 & $11,026,600$ & 137,300 & $7,663,350$ & $7,800,650$ & 0 & 0 & $4,015,909$ & 1 & Alarm \\
\hline May-07 & $14,746,400$ & $2,678,100$ & $12,475,850$ & $15,153,950$ & $2,641,982$ & 1 & 678,518 & 2 & OK \\
\hline Jun-07 & $13,367,400$ & $2,122,100$ & $12,554,200$ & $14,676,300$ & $4,806,314$ & 2 & 0 & 0 & Alarm \\
\hline Jul-07 & $15,026,000$ & $1,635,400$ & $12,764,350$ & $14,399,750$ & $6,694,095$ & 3 & 0 & 0 & Alarm \\
\hline Aug-07 & $15,641,100$ & $1,595,400$ & $13,745,150$ & $15,340,550$ & $9,522,677$ & 4 & 0 & 0 & Alarm \\
\hline Sep-07 & $15,812,700$ & $2,450,500$ & $13,419,650$ & $15,870,150$ & $12,880,859$ & 5 & 0 & 0 & Alarm \\
\hline
\end{tabular}

\section{Conclusion}

This research paper was about the problem of flow meter drift and how it may be detected. A background about this problem was given and the importance of it was stressed. Many previous works related to this problem were mentioned and discussed. The approach taken in this research to detect flow meter drift was statistical in nature. The benefits of this approach over other alternatives were 
explained. Of the plethora of statistical methods available, the method of the tabular CUSUM was chosen. The data available, however, can not be directly processed by this method. The concept of the virtual mean was introduced to overcome this obstacle. The virtual mean concept transformed the seasonal time series data to a medium that can be processed by the tabular CUSUM method. Several case studies where the method was applied were presented. The approach for detecting flow meter drift presented in this paper is not without limitations and shortcomings.

\section{References}

[1] Nilsson, U. R. C. (1998). A new method for finding inaccurate gas flow meters using billing data: Finding faulty meters using billing data. Flow Measurement and Instrumentation, 9(?), 237-242.

[2] Johnson, P. V. (1996). Unaccounted-For Water Puzzle: More Than Just Leakage. FLORIDA WATER RESOURCES JOURNAL.

[3] Hopkins, D., Savage, P. F., \& Foxt, E. (1995). Problems encountered during research into flow rate, pattern of water consumption and unaccounted-for water losses in urban areas. Flow Measurement and Instrumentation, 6(3), 173-179.

[4] Herwig, J., Keese, D., Rackebrandt, K. H., \& Schwiderski, H. W. (1994). Magnetic flowmeter with fault detection In U. S. P. Office (Ed.) (G01F 100 ed.). U.S.A: Fischer \& Porter Company.

[5] Keech, R. (2005). Flowmeter Fault Detection. In U. S. P. Office (Ed.) (G01F 160 ed., Vol. 6,843,137): ABB Limited.

[6] Han, D., \& Tsung, F. (2006). A Reference-Free Cuscore Chart of Dynamic Mean Change Detection and a Unified Framework for Charting Performance Comparison. Journal of the American Statistical Association, 101(473), 368-386.

[7] Page, E. S. (1954). Continuous Inspection Schemes. Biometrika, 41(1/2), 100-115.

[8] Zhao, Y., Tsung, F., \& Wang, Z. (2005). Dual CUSUM control schemes for detecting a range of mean shifts. IIE Transactions, 37(11), 1047-1057.

[9] Han, D., Tsung, F., \& Li, Y. (2007). A Cusum Chart with Local Signal Amplification for Detecting a Range of Unknown Shifts. International Journal of Reliability, Quality and Safety Engineering, 14(2), 81-97.

[10] Han, D., Tsung, F., Hu, X., \& Wang, K. (2007). Cusum and EWMA MultiCharts for Detecting a Range of Mean Shifts. Statistica Sinica, 17(3), 11391164.

[11] Han, D., \& Tsung, F. (2007). Detection and Diagnosis of Unknown Abrupt Changes Using CUSUM Multi-Chart Schemes. Sequential Analysis, 26(3), 225-249.

[12] Montgomery, D. C. (2005). Introduction to Statistical Quality Control: John Wiley \&Sons. Inc. 